\title{
Development of a Swedish Version of the Scale of Ethnocultural Empathy
}

\author{
Chato Rasoal $^{1 *}$, Tomas Jungert ${ }^{2}$, Stephan Hau ${ }^{3}$, Gerhard Andersson ${ }^{1}$ \\ ${ }^{1}$ Department of Behavioural Sciences and Learning, Linköping University, Linköping, Sweden; \\ ${ }^{2}$ Department of Psychology, McGill University, Montreal, Canada; \\ ${ }^{3}$ Department of Psychology, Stockholm University, Stockholm, Sweden. \\ Email: *chato.rasoal@liu.se \\ Received May 20 $0^{\text {th }}, 2011$; revised July $3^{\text {rd }}$, 2011; accepted August $4^{\text {th }}, 2011$.
}

\begin{abstract}
The development and establishment of a Swedish translation of the Scale of Ethnocultural Empathy (SEE) was investigated in a sample of 788 participants. The SEE is a self-report instrument and is used to measure empathy directed toward people from ethnic cultural groups who are different from the respondent's own ethnocultural group. Principal components factor analyses and confirmatory factor analyses (CFA) provided evidence for a four-factor structure. The factors were Acceptance of Cultural Differences, Communicative Ethnocultural Empathy, Ethnocultural Empathic Awareness, and Intellectual Ethnocultural Empathy. The factors were moderately intercorrelated, and additional correlational analyses showed convergent validity in high correlations between the four factors and the two subscales Empathic Concern and Perspective Taking of the Interpersonal Reactivity Index (IRI). Possible applications of this scale in a healthcare context are discussed.
\end{abstract}

Keywords: Ethnocultural Empathy, Confirmatory Factor Analysis, Empathy, Explorative Factor Analysis

The word empathy comes from the Greek word empathiea, which means sensing other people's reactions and entering their world (Campbell \& Babrow, 2004). Empathy is a profound human characteristic and a fundamental feature of helping behaviour. In the psychological literature, empathy as a personality trait and as a learned ability has been assessed by quantitative measures (Davis, 1996; Hogan, 1969; Mehrabian \& Epstein, 1972). The ability to empathize and identify with others is essential to all human relationships and can be understood as a bond that makes social life possible (Hogan, 1969; Eisenberg \& Strayer, 1987). Empathic individuals have a better capacity to orientate themselves in a variety of interpersonal relationships in their lives, in professional life, and in family life as well as in contact with people from other cultural and ethnic groups (Batson \& Ahmed, 2001; Hoffman, 2000).

Ethnocultural empathy is defined as empathy directed towards people from racial and ethnic cultural groups who are different from one's own ethnocultural group, and is a relatively new concept that has not yet become established in the psychological literature and theory. Wang et al. (2003) coined the term ethnocultural empathy from theories on general and cultural empathy, and operationalized the concept by developing a scale of ethnocultural empathy. According to them, the Scale of Ethnocultural Empathy (SEE) is the first measure that provides support for the theoretical construct of empathy in multicultural settings. Furthermore, they assume that it is important to identify measures and afterwards find ways to develop the ability of ethnocultural empathy between different ethnic groups in order to reduce conflicts and to increase the level of tolerance, openness, and respect in human relations. According to Wang et al. (2003), ethnocultural empathy involves four components. 1) Communicative empathy (empathic feeling and expression), which focuses on concern about communication of discriminatory or prejudiced attitudes or beliefs as well as emotional or affective responses to the emotion and/or experiences of people from ethnic groups different from one's own. 2) Intellectual empathy, which means the ability to understand the experiences and emotions of people from dif- ferent ethnic backgrounds by trying to take their perspective in viewing the world. 3) Ethnocultural empathetic awareness, which focuses on the awareness or knowledge that one has about the experiences of people from ethnic groups different from one's own. This awareness of the emotions and experiences of others is particularly related to their experiences of discrimination or the unequal treatment of different groups. 4) Acceptance of cultural differences, which means accepting, understanding, and valuing the cultural traditions and norms of those with a different background (e.g. understanding why people from other ethnic groups like to dress in traditional clothes or speak in their own language). Wang et al. (2003) based the SEE on these four components in order to measure the extent of ethnocultural empathy. The SEE shows acceptable internal consistency and construct validity. Test-retest reliability obtained from the US sample was high (Cronbachs alpha $=0.91$ ). In fact, SEE is the first empirical measure to investigate cultural empathy in multicultural relations (Wang et al., 2003). To our knowledge the SEE has not been translated and used in other countries, which would provide further support for the construct and facilitate cross-cultural research.

Swedish society is becoming more ethnically and culturally diverse (Daun, 1998; Ekblad, Janson \& Svensson, 1996; Mlekov \& Widell, 2003; Rasoal, 2009; Rasoal, Eklund \& Hansen, 2010; Rasoal, Jungert, Hau, Stiwne \& Andersson, 2009; Svanberg \& Runblom, 1989). For a long time, Sweden was a country with a relatively homogeneous population of Germanic origin, whereas currently approximately $15 \%$ of the population has a non-Swedish origin (Statistics Sweden, 2005). According to the Swedish Integration Board, it is not uncommon for individuals of other ethnic groups than Swedish to feel discriminated by different public authorities, private companies, and other institutions, such as schools (Integration Board, 2005). The findings mentioned above imply that Swedish men and women, who are actively working in Swedish authorities, companies, and institutions, may show less cultural empathy towards other ethnic groups than their own. In addition, they may be less open and show less respect and tolerance for these 
groups. By deliberately showing empathy individuals might increase their capacity to identify and understand other individuals' intellectual states, situations, feelings, and motives (Chang \& Bemak, 2002; Ivey, Ivey \& Simek-Downing, 1993; Kuhn, 2001). Batson et al. (1997) showed that when an empathic perspective was established by induction, this was effective in changing individuals' attitudes towards stigmatized groups. However measuring empathy in general would not adequately portray an individual's empathy towards persons who are culturally different (Wang et al., 2003).

In the present study we translated and used the SEE in a sample of undergraduate students. The aim was to develop and establish a Swedish version of the SEE and to compare its internal structure and convergent validity with other constructs which measure basic empathy. Developing a SEE for Swedish settings could give a valuable boost to better understanding of the structure of cultural empathy and thereby make it possible to measure this ability and apply it in different contexts (e.g. institutions, healthcare system, etc.) where people come into contact with individuals from other ethnic backgrounds.

\section{Methods}

\section{Participants and Procedures}

A sample of 799 undergraduate students was drawn from courses and programmes at Linköping University and secondary schools in Sweden. Measures were administered in person (11 missing or invalid). The data set used for this analysis consisted of 788 observations. Participants completed the scale in large groups of 30 to 60 students. There were 553 female (70\%) and 235 male (30\%) secondary and undergraduate students. Their ages ranged from 15 to $47(\mathrm{M}=24.3, \mathrm{SD}=5.8)$. Most of the respondents described themselves as ethnic Swedish $(92.8 \%$, $\mathrm{N}=731$ ), 1.4\% were ethnic Bosnian, 1.1\% were ethnic Finnish, and the remainder were from other ethnic backgrounds. In the questionnaire package we asked the participants about their gender, age, and ethnicity. Participants were assured of their anonymity and were informed that they were free to withdraw from the study at any time. Approximately 15 to 20 minutes were needed to complete the questionnaire package.

\section{Instruments}

The questionnaire package used in this study consisted of four parts: a questionnaire on demographics, the SEE, and the two subscales of the Interpersonal Reactivity Index, i.e. Empathic concern, and Perspective-taking (IRI: Davis, 1996). The SEE is a 31-item forced choice self-report measure that generates a total score and four subscale scores (Wang et al., 2003). The subscales reported by the developers of the SEE reflected the following factor structures: factor 1, Empathic Feeling and Expression (EFE),_consisted of 15 items (e.g. 'I share the anger of those who face injustice because of their racial and ethnic backgrounds'); factor 2, Ethnocultural Empathy Awareness (EA), consisted of four items (e.g. 'I feel irritated when people of different racial or ethnic background speak their language around me'); factor 3, Acceptance of Cultural Differences (AC), consisted of five items (e.g. 'I am aware of institutional barriers [e.g. restricted opportunities for job promotion] that discriminate against racial or ethnic groups other than my own') and factor 4, Empathy Perspective-Taking (EP), consisted of seven items (e.g. 'It is easy for me to understand what it would feel like to be a person of another racial or ethnic background other than my own'). In addition, respondents also completed the two subscales of Interpersonal Reactivity Index (IRI: Davis, 1996) Empathic concern (seven items), and Perspective-taking (seven items) designed to tap two separate dimensions on basic empathy (Davis, 1996). All of the items were listed in random order and rated on a five-point Likert-type scale $(1=$ strongly disagree to $5=$ strongly agree). The items were phrased both positively and negatively to offset any potential response bias. Negatively phrased items were reverse-scored. The questionnaire package consisted of 45 items in total. Scores for the SEE were obtained by adding up the item scores. Higher scores indicated a higher level of ethnocultural empathy. Integrity of the Swedish translation was verified by the back translation technique. Discrepancies in meaning from the original English version were noted and the Swedish translation was adjusted by an assistant professor in social psychology and by experts fluent in both English and Swedish.

\section{Results}

Factor loadings, means, and standard deviations of the four factor solutions are presented in Table 1.

\section{Confirmatory Factor Analysis (CFA)}

An initial CFA was used to test if the four-factor structure specified as the four subscales of the SEE (Wang et al., 2003) would be obtained. We used EQS 6.1 (Byrne, 2006) to specify the expected factor loadings of the 31 items. The results showed that these factor loadings failed to validate the original version of the SEE. Thus, to determine the number of factors, a principal components analysis with an oblique rotation was conducted. We used a scree plot in PASW statistics 18, which suggested retaining four components as in the original version of the SEE. Moreover, we used the following criteria: four components with eigenvalues of greater than 1.00 were retained and items possessing factor loadings of 0.40 or greater were assigned to each factor. This analysis showed that two items did not load on any of the four factors. These two items were items 2 ('I don't know a lot of information about important social and political events of racial and ethnic groups other than my own') and 4 ('I know what it feels like to be the only person of a certain race or ethnicity in a group of people') of the original version of the SEE. They were consequently removed from further analyses. An additional principal components analysis of the remaining 29 items was chosen because it resulted in the most sound factor loadings. The factor structure had four components with eigenvalues greater than 1.00 and all items possessed factor loadings of 0.40 or greater. The total variance of these four factors was $52 \%$.

A second CFA was then used to specify the factor loadings obtained in the final principal components analysis. The analysis was twofold, examining the adequacy of the exploratory principal components analysis and assessing whether this model would provide a good fit with the data. A maximum likelihood method and robust independence model in the EQS program were used to estimate goodness of fit of the new four-factor model. In this analysis seven indices were used to assess the goodness of fit of the models: Satorra-Bentler scaled chi-square, chi-square/df ratio, comparative fit index (CFI; Hu $\&$ Bentler, 1999; best if 0.90 or greater), Bollen's fit index (IFI) and root-mean-square error of approximation (RMSEA; Browne 
Table 1.

Factor loadings, item means, and standard deviations of the Swedish version of SEE.

\begin{tabular}{|c|c|c|c|}
\hline Item & Factor loading & M & SD \\
\hline \multicolumn{4}{|l|}{ Factor 1: Acceptance of Cultural Differences } \\
\hline $\begin{array}{l}\text { 5. I get impatient when communicating with people from other racial or ethnic backgrounds, regardless of how well } \\
\text { they speak English. }\end{array}$ & .90 & 3.32 & 1.64 \\
\hline 21. I don't care if people make racist statements against other racial or ethnic groups. & .89 & 3.36 & 1.57 \\
\hline 26. I share the anger of people who are victims of hate crimes (e.g. intentional violence because of race or ethnicity). & .89 & 3.22 & 1.57 \\
\hline 15. I get disturbed when other people experience misfortunes owing to their racial or ethnic background. & .87 & 3.28 & 1.42 \\
\hline 8. I don't understand why people of different racial or ethnic backgrounds enjoy wearing traditional clothing. & .82 & 3.31 & 1.44 \\
\hline 1. I feel annoyed when people do not speak standard Swedish. & .82 & 3.30 & 1.50 \\
\hline $\begin{array}{l}\text { 7. I am aware of institutional barriers (e.g. restricted opportunities for job promotion) that discriminate against racial or } \\
\text { ethnic groups other than my own. }\end{array}$ & .71 & 3.11 & 1.30 \\
\hline 16. I rarely think about the impact of a racist or ethnic joke on the feelings of people who are targeted. & .66 & 3.23 & 1.32 \\
\hline 10. I feel irritated when people of different racial or ethnic background speak their language around me & .63 & 3.20 & 1.35 \\
\hline 17. I am not likely to participate in events that promote equal rights for people of all racial and ethnic backgrounds. & .63 & 3.15 & 1.30 \\
\hline 20. I can see how other racial or ethnic groups are systematically oppressed in our society. & .62 & 3.13 & 1.10 \\
\hline \multicolumn{4}{|l|}{ Factor 2: Communicative Ethnocultural Empathy } \\
\hline 12. I share the anger of those who face injustice because of their racial and ethnic backgrounds. & .70 & 3.84 & 0.97 \\
\hline 18. I express my concern about discrimination to people from other racial or ethnic groups. & .66 & 3.43 & 0.92 \\
\hline $\begin{array}{l}\text { 22. When I see people who come from a different racial or ethnic background succeed in the public arena, I share their } \\
\text { pride. }\end{array}$ & .58 & 4.10 & 0.83 \\
\hline 3. I am touched by films or books about discrimination issues faced by racial or ethnic groups other than my own. & .56 & 3.91 & 0.99 \\
\hline 13. When I interact with people from other racial or ethnic backgrounds, I show my appreciation of their cultural norms. & .53 & 3.46 & 0.87 \\
\hline 11. When I know my friends are treated unfairly because of their racial or ethnic backgrounds, I speak up for them. & .47 & 4.23 & 0.77 \\
\hline $\begin{array}{l}\text { 30. When I hear people make racist jokes, I tell them I am offended even though they are not referring to my racial or } \\
\text { ethnic group. }\end{array}$ & .46 & 2.86 & 1.15 \\
\hline \multicolumn{4}{|l|}{ Factor 3: Ethnocultural Empathic Awareness } \\
\hline 25. I am aware of how society differentially treats racial or ethnic groups other than my own. & .66 & 4.23 & 0.82 \\
\hline 24. I recognize that the media often portray people based on racial or ethnic stereotypes. & .57 & 3.99 & 0.91 \\
\hline $\begin{array}{l}\text { 6. I can relate to the frustration that some people feel about having fewer opportunities owing to their racial or ethnic } \\
\text { backgrounds. }\end{array}$ & .53 & 4.20 & 0.89 \\
\hline $\begin{array}{l}\text { 27. I do not understand why people want to keep their indigenous racial or ethnic cultural traditions instead of trying to } \\
\text { fit into the mainstream. }\end{array}$ & .44 & 3.87 & 1.04 \\
\hline \multicolumn{4}{|l|}{ Factor 4: Intellectual Ethnocultural Empathy } \\
\hline 28. It is difficult for me to put myself in the shoes of someone who is racially and/or ethnically different from me. & .72 & 3.29 & 1.06 \\
\hline $\begin{array}{l}\text { 19. It is easy for me to understand what it would feel like to be a person of a racial or ethnic background other than my } \\
\text { own. }\end{array}$ & .71 & 3.01 & 1.15 \\
\hline $\begin{array}{l}\text { 31. It is difficult for me to relate to stories in which people talk about the racial or ethnic discrimination they experience } \\
\text { in their everyday lives. }\end{array}$ & .58 & 3.33 & 1.04 \\
\hline
\end{tabular}

$\mathrm{N}=788$; there are 25 items.

\& Cudeck, 1993; best if 0.05 or less). A consequence of the first CFA based on the 29 items was that four additional items were deleted because the model did not provide a good fit with the data owing to excessively weak loadings of these four items. These deleted items were items 9, 14, 23, and 29 of the original SEE. A final four-factor solution was obtained (see Table 1).
The variance of the factors was initially fixed at 1.0. The model had good fit with the data, as indicated by the following indices: Satorra-Bentler scaled $\chi_{290}^{2}=(\mathrm{N}=788)=742, p<.001$ $\chi^{2} / \mathrm{df}$ ratio $=2.5 ; \mathrm{NNFI}=0.94 ; \mathrm{NFI}=0.92 ; \mathrm{CFI}=0.95 ; \mathrm{IFI}=$ 0.95 ; RMSEA $=0.05$. The first factor Acceptance of Cultural Differences (11 items) accounted for $28 \%$ of the total variance 
(eigenvalue $=7.1$ ). The second factor Communicative Ethnocultural Empathy (seven items) accounted for $16 \%$ of the total variance (eigenvalue $=4.0$ ). The third factor Ethnocultural Empathic Awareness (four items) accounted for $6 \%$ of the total variance (eigenvalue $=1.6$ ), and the last factor Intellectual Ethnocultural Empathy (three items) accounted for 5\% of the total variance (eigenvalue $=1.2$ ). These four factors are specified as the four subscales of the SEE (Wang et al., 2003).

To measure internal consistency, we calculated Cronbach's $\alpha$ coefficients (see Table 2). The Cronbachs $\alpha$ coefficient for the Swedish version of the SEE subscales was $0.94,0.76,0.62$, and 0.71 , and for the total SEE it was 0.88 , whereas the Cronbachs $\alpha$ coefficient for the US version of the SEE subscales was 0.89 , $0.75,0.73$, and 0.76 , and for the total SEE the coefficient was 0.91 . Both the Swedish and the US versions of the SEE showed acceptable levels of internal consistency (Table 3 ).

\section{Convergent Validity}

To measure convergent validity correlation analyses were performed on each of the four factors and the total SEE scale scores with the Empathic concern and Perspective-taking subscales of the Interpersonal Reactivity Index (Davis, 1996). The intercorrelations between the factors suggest that the four factors are somewhat interrelated but still represent distinct constructs. There were significant correlations in the low to moderate range for IRI subscales and each factor of the SEE and the total SEE score (see Table 3).

\section{Discussion}

The aim of the study was to translate the US version of the Scale of Ethnocultural Empathy (SEE) and develop and establish a Swedish version. We were interested in determining the factor solution of a Swedish version of SEE that had the best goodness of fit with the data, and investigating whether the SEE is associated with the two subscales Empathic concern and Perspective-taking of the IRI. We also examined the internal consistency, and discriminate as well as convergent validity of the new version of the SEE.

The Swedish factor solution revealed four distinct factors underlying the scores of the SEE. This four-factor solution was chosen because it resulted in the most robust factor structure with stronger item loadings and factor internal consistency and was the most conceptually interpretable factor structure. The US version also offered a four-factor solution, but the item loadings on the factors of the Swedish version were somewhat different and were adapted to the Swedish context in terms of the history of immigration, culture, policies on immigration and integration, and language.

Out of the 31 items of the original SEE, six items were eliminated from the Swedish version. Items 2 and 4 were deleted. There were three main arguments for deleting them. First, these two items loaded as a fifth factor of the exploratory principal components analysis despite the indication by the scree plot that four factors were the most appropriate and meaningful

Table 2.

Subscale means, standard deviations and alpha level for US and Swedish versions of SEE.

\begin{tabular}{|c|c|c|c|c|c|c|}
\hline \multirow[b]{2}{*}{ Factor } & \multicolumn{3}{|c|}{ Swedish } & \multicolumn{3}{|c|}{ US } \\
\hline & Alpha $\alpha$ & $\mathbf{M}$ & SD & Alpha $\alpha$ & $\mathbf{M}$ & SD \\
\hline 1 Empathic Feeling and Expression & .76 & 3.7 & .60 & .89 & 4.3 & 0.86 \\
\hline 2 Empathic Perspective-Taking & .71 & 3.2 & .86 & .75 & 3.4 & 1.0 \\
\hline 3 Acceptance of Cultural Differences & .94 & 3.2 & 1.13 & .73 & 4.6 & 0.98 \\
\hline 4 Empathic Awareness & .62 & 4.1 & .63 & .76 & 4.6 & 0.99 \\
\hline 5 SEE Total & .88 & 3.5 & .60 & .91 & 4.2 & 0.75 \\
\hline
\end{tabular}

Note: The US study was conducted by Wang et al. (2003).

Table 3.

Intercorrelations between subscale and total scores of Swedish version of SEE, Interpersonal Reactivity Index (IRI).

\begin{tabular}{|c|c|c|c|c|c|c|c|}
\hline Measure & 1 & 2 & 3 & 4 & 5 & 6 & 7 \\
\hline 1 (Acceptance of Cultural Differences) & --- & .09 & 04 & $.12 * *$ & $.88 * *$ & $.78 * *$ & $.42 * *$ \\
\hline 2 (Communicative Ethnocultural Empathy) & & --- & $.49^{* *}$ & $.35^{* *}$ & $.38 * *$ & $.27 * *$ & $.32 * *$ \\
\hline 3 (Ethnocultural Empathic Awareness) & & & --- & .24 & $.38 * *$ & $.18^{* *}$ & $.29 * *$ \\
\hline 5 (SEE Total) & & & & & --- & $.78^{* *}$ & $.55 * *$ \\
\hline 6 (IRI Empathic Concern) & & & & & & --- & $.44^{*}$ \\
\hline 7 (IRI Perspective-Taking) & & & & & & & \\
\hline
\end{tabular}

Note: SEE = Scale of Ethnocultural Empathy; Subscales of IRI = Davis Interpersonal Reactivity Index. 
solution. Second, to define a factor as only two items is not acceptable. Third, these two items were the only ones that were phrased as 'I know' and it is possible that Swedish respondents are more cautious about agreeing with such statements than US respondents. A Swedish respondent who is highly empathetic and/or is aware of the discrimination that occurs in society would not claim to know what it is like to belong to an ethnic minority, because of the Sweden notion that you should be careful about what you claim to know. An additional four items $(9,14,23$, and 29) were eliminated when the principal components analysis was confirmed in EQS because they did not contribute to an interpretable and strong factor structure with goodness of fit with the data.

The four factors of the established Swedish SEE are Acceptance of Cultural Differences (Factor 1) with 11 items (e.g. 'I get impatient when communicating with people from other racial or ethnic backgrounds, regardless of how well they speak English'), Communicative Ethnocultural Empathy (Factor 2) with seven items (e.g. 'I express my concern about discrimination to people from other racial or ethnic groups'), Ethnocultural Empathic Awareness (Factor 3) with four items (e.g. 'I am aware of how society differentially treats racial or ethnic groups other than my own'), Intellectual Ethnocultural Empathy (Factor 4) with three items (e.g. 'It is difficult for me to put myself in the shoes of someone who is racially and/or ethnically different from me')

The first factor, Acceptance of cultural differences, is composed of items that indicate acceptance, understanding and valuing of cultural traditions and customs of individuals from different racial and ethnic groups. Some items also concern feelings that relate to individuals belonging to other ethnic groups. Most of these items belonged to the subscale Acceptance of Cultural Differences of the original version of SEE, but five of the items, which pertain to feelings, belonged to the subscale Empathic Feeling and Expression of the original SEE. One possible explanation for this could be that Swedish respondents may have regarded those items as less emotive and that in the Swedish version the awareness aspect is more salient than the emotional aspect.

The second factor Communicative Ethnocultural Empathy is concerned with the communication of behaviour that reflects prejudice and discrimination. These items also pertain to actions that are taken to prevent discriminatory behaviour or defend ethnic minorities, as in 'I show my appreciation of....' and 'I speak up for...'. This factor only includes items that belonged to the subscale Empathic Feeling and Expression of the original SEE. A possible explanation for this similarity may be that there is not as much room for cultural interpretation of this factor, which pertains to thoughts, feelings, and actions directly related to ethnic discrimination.

Factor 3, Ethnocultural Empathic Awareness, is about awareness and knowledge about what it might be like to belong to an ethnic minority different from one's own. Three of the four items belonging to this factor are clearly about awareness and knowledge about experiences of discrimination and prejudice against people from other ethnic groups, and loaded on the same factor in the original version of the SEE. The last item, however, refers more to perspective-taking than to awareness.

The last factor, Intellectual Ethnocultural Empathy, includes items that deal with one's efforts to understand what it is like to belong to an ethnic minority, such as trying to put oneself in the shoes of another person. All of these items belong to the subscale Empathic Perspective-Taking of the original SEE. This similarity may also be because there are few cultural differ- ences between the US and the Swedish culture regarding a person's efforts to understand what it is like to belong to an ethnic minority. The results from the exploratory factor analysis suggest that the concept of ethnocultural empathy can be measured in Sweden. The CFA replicated the original findings. It is also possible that cultural issues may have an impact on the results. Finally, the first CFA presented by Wang et al. (2003) was not reliable and a higher order hierarchal model was used instead.

The intercorrelations between the four factors of the Swedish version of SEE varied from very low to moderate. The correlations between the second, third and fourth factors were significant and stronger than the correlations between the first factor and the other factors. The best explanation for this is probably that the first factor, Acceptance of Cultural Differences, includes 11 items that deal more with knowledge and experience of diverse cultures and acceptance of this. In other words, this factor taps a broader span of what Ethnocultural Empathy is and works on many levels. There seems to be more room for interpretations regarding the items of this factor, i.e. what is perceived as empathy among our respondents. This factor had a significant, albeit small correlation with Intellectual Ethnocultural Empathy. The other three factors are more precise and concrete, which leaves less room for interpretation. Thus, they have stronger correlations among themselves. The intercorrelations between each factor and the total SEE were all significant with moderate to strong correlations.

We also found evidence of convergent validity for the SEE scale and its four factors. Significant correlations in the low to moderate range were found between the subscales of IRI (Perspective-Taking and Empathic Concern) and the sub-factors of SEE as well as with the total score of SEE. The Acceptance of Cultural Differences subscale had the highest correlation coefficient with the IRI subscales. The most probable explanation is that this factor contains the greatest number of items of the Swedish SEE, and thus taps the essence of basic empathy. A possible explanation for the differences between the US and the Swedish version of the SEE is that cultural differences between the Swedish and US society exist. Another possible explanation could be that differences came about because of different samples, e.g. the distribution of men/women or educational profiles. It could also be that the capacity of Ethnocultural Empathy develops later in life and is not equally present in undergraduate students and graduates or adults with greater experience of working life.

There are some limitations of the study. First we relied on self-report measures and although this facilitates the collection of large data sets, we are aware that self-report does not always transfer to actual behaviour, in this case behaviours that would corroborate the concept of ethnocultural empathy (Fan et al., 2006). A second limitation of the present study might be the primary use of undergraduate students and secondary school students in the locality of the university. Obviously, these students may not be representative of all individuals in Sweden. Clark and Watson (1995) stressed the importance of examining the factor structure of psychological assessment scales in heterogeneous samples. A future task would be to test the SEE in a more diverse group (e.g., socioeconomic status, ethnicity, age, etc.). Such data would be required to establish norms as well. An additional future task would be to add items to the Swedish version of the SEE that may better reflect Ethnocultural Empathy in a Swedish context.

To conclude, the findings from this study indicate that the Swedish version of the SEE is a reliable and valid measure of empathy directed towards people from ethnic cultural groups 
who are different from one's own ethnocultural group. The next steps will be to add items that may reflect a Swedish context even better and to use the questionnaire in different student courses like medicine, psychology, and social worker programmes and to apply the SEE in different contexts (e.g. institutions, healthcare system, etc.) where people are in contact with individuals from other ethnic backgrounds.

\section{Acknowledgements}

We would like to thank Helena Dedic for her valuable suggestions regarding statistical analyses.

\section{References}

Batson, C. D., \& Ahmed, N. (2001). Empathy-induced altruism in a prisoner's dilemma II: What if the target of empathy has defected? European Journal of Social Psychology, 73, 517-522.

Batson, C. D., Polycarpou, M. P., Harmon-Jones, E., Imhoff, H. J., Mitchener, E.C., Bednar, L. L., et al. (1997). Empathy and attitudes: Can feeling for members of a stigmatized group improve feelings toward the group? Journal of Personality and Social Psychology, 72, 105-118. doi:10.1037/0022-3514.72.1.105

Bentler, P. M. (1995). EQS structural equations program manual. Encino, CA: Multivariate Software.

Browne, M. W., \& Cudeck, R. (1993). Alternative ways of assessing model fit. In K. A. Bollen and J. S. Long (Eds.), Testing structural equation models (pp. 136-162). Newbury Park, CA: Sage.

Byrne, B. M. (2006). Structural equation modeling with EQS and EQS/Windows: Basic concepts, application and programming. Mahwah, NJ: Lawrence Erlbaum Associates.

Campbell, R. G., \& Babrow, A. S. (2004). The role of empathy in responses to persuasive risk communication: Overcoming resistance to HIV prevention messages. Health Communication, 16, 159-182. doi:10.1207/S15327027HC1602 2

Chung, R. C. -Y., \& Bemak, F. (2002). The relationship of culture and empathy in cross-cultural counseling. Journal of Counseling and Development, 80, 154-159.

Clark, L. A., \& Watson, D. (1995). Constructing validity: Basic issues in objective scale development. Psychological Assessment, 7, 309319. doi:10.1037/1040-3590.7.3.309

Daun, A. (1998). Swedish mentality (3rd ed.). Stockholm: Rabén Prisma.

Davis, H. M. (1996). Empathy: A social psychological approach. Colorado: Westerview Press.

Eisenberg, N., \& Strayer, J. (1987). Empathy and its development. New York: Cambridge University Press.
Ekblad S., Janson S., \& Svensson P.-G. (1996). Encounter in healthcare. Transcultural perspective on healthcare. Stockholm: Liber Utbildning.

Fan, X., Miller, B. C., Park, K. E., Winward, B. W., Christensen, M. Grotevant, H. D., \& Tai, R. H. (2006). An exploratory study about inaccuracy and invalidity in adolescent self-report surveys. Field Methods, 18, 1-22. doi: $10.1177 / 152822$ X06289161

Hoffman, M. L. (2000). Empathy and moral development. New York: Cambridge University Press.

Hogan, R. (1969). Development of an empathy scale. Journal of Consulting and Clinical Psychology, 33, 307-316. doi:10.1037/h0027580

Hu, L., \& Bentler, P. M. (1999). Cutoff criteria for fit indexes in covariance structure analysis: conventional criteria versus new alternatives. Structural Equation Modeling, 6, 1-55. doi:10.1080/10705519909540118

Integration Board (Sweden). (2005). Report Integration. Malmö: Elanders Berlings.

Ivey, A. E., Ivey, M. B., \& Simek-Downing, L. (1993). Counseling and psychotherapy: integrating skills, theory, and practice. Englewood Cliffs, NJ: Prentice Hall.

Kuhn, J. L. (2001). Toward an ecological humanistic psychology. Journal of Humanistic Psychology, 41, 9-24. doi: $10.1177 / 0022167801412003$

Mehrabian, A., \& Epstein, N. (1972). A measure of emotional empathy. Journal of Personality, 40, 525-543. doi:10.1111/j.1467-6494.1972.tb00078.x

Mlekov, K., \& Widell, G. (2003). How do we encounter diversity in the workplace? Lund: Studentlitteratur.

Rasoal, C. (2009). Ethnocultural empathy: Measurement, psychometric properties, and differences between students in healthcare educations (Doctoral Dissertation). Linköping University. Linköping Studies in Behavioral Sciences No: 147.

Rasoal, C., Eklund, J., \& Hansen, E. (2011). Toward a conceptualization of ethnocultural empathy. Journal of Social, Evolutionary, and Cultural Psychology, 5, 1-13.

Rasoal, C., Jungert. T., Hau, S., Edvardsson-Stiwne, E., \& Andersson, G. (2009). Ethnocultural empathy among students in healthcare education. Evaluation \& the Health Professions, 3, 300-313. doi: $10.1177 / 0163278709338569$

Statistics Sweden. (2005). Resident population estimates of the Sweden by sex, age, nationality, and mortality, 1960-2005. URL (Retrieved October 2006) http://www.scb.se/statistik/BE/BE0101/2006M01/be 0101 tab8samdrag.xls

Svanberg, I., \& Runblom, H. (1989). Multicultural Sweden. A handbook about ethnic groups and minorities. Stockholm: Gildlunds.

Wang, Y. W., Bleier, J., Davidson, M., Savoy, H., Tan, J., \& Yakushko, O. (2003). The scale of ethnocultural empathy. Development, validation, and reliability. Journal of Counselling Psychology, 2, 221-234. doi: $10.1037 / 0022-0167.50 .2 .221$ 